\title{
FRACTIONAL PATH INTEGRATION FOR GROWTH DYNAMICS AND SCALING BEHAVIOR IN COMPLEX SYSTEMS
}

\author{
CHRISTOPER C. BERNIDO \\ Research Center for Theoretical Physics, Central Visayan Institute Foundation, \\ Jagna, Bohol 6308, Philippines \\ E-mail: cbernido@mozcom.com \\ MATTHEW G. O. ESCOBIDO \\ W. Sycip Graduate School of Business, Asian Institute of Management, \\ Makati City, Philippines \\ E-mail: MEscobido@AIM.EDU \\ M. VICTORIA CARPIO-BERNIDO \\ Research Center for Theoretical Physics, Central Visayan Institute Foundation, \\ Jagna, Bohol 6308, Philippines \\ E-mail: cbernido@mozcom.com
}

\begin{abstract}
An analytical model for growth rates in complex systems based on the sum-over-allhistories approach is presented. To endow the rate of growth with memory of the past, fractional Brownian paths are used. A probability distribution function and its Laplace transform are evaluated and compared with an empirical formula derived from data of selected complex systems. Scaling behavior of these systems is also discussed.
\end{abstract}

\section{Introduction}

Complex systems exhibit fluctuations arising from nonlinear interactions among multiple components with large numbers of degrees of freedom. The observed fluctuations of macroscopic quantities of a complex system, however, could display regularities in behavior. This compels us to look for general principles and universal features, in spite of not knowing detailed descriptions of multiple interactions among components of the system. For example, in spite of the seeming randomness of fluctuations in a complex system, short-term and long-term memory in its dynamics could account for regularities in behavior. In this paper, we incorporate memory in the growth dynamics of a complex system by using fractional Brownian motion to characterize rate fluctuations of a given macroscopic variable. This is presented in section 3. The sum-over-all-histories approach is then applied in section 4 to obtain a probability distribution function. Next, we discuss in section 5 the self-similar behavior and scaling property of the model. In sections 6 and 7, we compare analytical results with an empirical formula obtained from several complex 
systems, in particular: (a) the growth rates of companies of different sizes and products manufactured $^{1}$; (b) the metabolic rate of living organisms (rate at which an animal consumes oxygen $)^{2}$; and (c) growth rates of new words as language evolves ${ }^{3}$. We give concluding remarks in section 8 .

\section{Growth Fluctuations without Memory}

Consider a complex system characterized by a macroscopic quantity, labelled $S$, which fluctuates in time. For example, $S$ could be the oxygen consumption of an animal at a given time. To track the decrease or increase of $S$ in time, we may start by looking at the simplest model of growth rates proposed by Gibrat ${ }^{4}$. One defines growth rate as, $R=S_{1} / S_{0}$, where $S_{0}$ and $S_{1}$ are values determined in two consecutive periods. Gibrat's law can then be used to relate growth rate at time $t$ and a prior period $t-1$ as,

$$
R_{t}-R_{t-1}=\varepsilon_{t} R_{t-1}
$$

where $\varepsilon_{t}$ is a random variable denoting the proportionate growth between the two periods. The uncorrelated random number $\varepsilon_{t}$ has a mean close to zero. Eq. (2.1) may be rewritten as,

$$
\begin{aligned}
R_{t} & =R_{t-1}\left(1+\varepsilon_{t}\right) \\
& =R_{t-2}\left(1+\varepsilon_{t-1}\right)\left(1+\varepsilon_{t}\right) \\
& =R_{0}\left(1+\varepsilon_{1}\right)\left(1+\varepsilon_{2}\right) \ldots\left(1+\varepsilon_{t}\right) .
\end{aligned}
$$

Considering short-time periods where $\varepsilon_{i}(i=1,2, \ldots, t)$ is small, we take the log of Eq. (2.2) to get,

$$
\ln \left(R_{t}\right) \simeq \ln R_{0}+\varepsilon_{1}+\varepsilon_{2} \ldots+\varepsilon_{t}
$$

where $\ln \left(1+\varepsilon_{i}\right) \simeq \varepsilon_{i}$. If we define, $r=\ln R=\ln \left(S_{1} / S_{0}\right)$, we get

$$
r_{t} \simeq r_{0}+\sum_{i=1}^{t} \varepsilon_{i}
$$

where displacements $\varepsilon_{1}, \varepsilon_{2}, \varepsilon_{3}, \ldots$, are assumed independent and identically distributed random variables. Eq. (2.4) then shows $r_{t}$ to be a simple random walk starting at $r_{0}$ with $t$ steps. One may also imagine this equation in analogy to a simple random walk on a periodic lattice where the length of each step is $\varepsilon$. At each step, the next jump may proceed toward any of the nearest-neighbor sites.

In the limit where the number of steps increases and the step sizes approach zero, one can pass over to a situation analogous to Eq. (2.4) but in continuous time using a Brownian motion $B(t)$ which starts at $r_{0}$ such that, 


$$
r(t)=r_{0}+B(t)
$$

with the expectation value, $\langle B(t)\rangle=0$. Eqs. (2.4) and (2.5), however, are Markovian which constrains applicability to real-world problems where short or long-memory processes may prevail in the evolution of complex systems. In the next section, we modify Eq. (2.5) by endowing growth rates of $S$ with memory using fractional Brownian motion $B^{H}(t)$, instead of simply $B(t)$.

\section{Growth Fluctuations with Memory}

Growth fluctuations due to a huge number of external and internal interactions could include possible correlations in time that can manifest as short-term or longterm memory. Hence, instead of Eq. (2.5) we consider,

$$
r(t)=r_{0}+B^{H}(t)
$$

where as before, $r(t)=\ln R_{t}$, with $R_{t}$ the growth rate at time $t$. The $B^{H}(t)$ is a fractional Brownian motion ( $\mathrm{fBm}$ ) which can accommodate correlations in time, or memory, defined by ${ }^{5}$,

$$
B^{H}(t)=\frac{1}{\Gamma\left(H+\frac{1}{2}\right)} \int_{0}^{t}\left(t-t^{\prime}\right)^{H-1 / 2} d B\left(t^{\prime}\right) .
$$

In Eq. (3.2), $H$ is the Hurst parameter where, $1 / 2<H<1$ describes enhanced diffusion exhibiting a long-memory property, and $0<H<1 / 2$ describes suppressed diffusion for short-memory processes ${ }^{5}$. We note that $B\left(t^{\prime}\right)$ is the ordinary Brownian motion whose time derivative is a Gaussian white noise, i.e., $\omega\left(t^{\prime}\right)=d B\left(t^{\prime}\right) / d t^{\prime}$. Eq. (3.2) can, therefore, be viewed as a white noise functional where, $d B\left(t^{\prime}\right)=$ $\omega\left(t^{\prime}\right) d t^{\prime}$.

\section{Sum-over-all Histories}

Given Eq. (3.1), one could consider an ensemble of fractional Brownian paths all of which start with a growth rate $r_{0}$ at time $t=0$. If, however, we are interested in those paths which end at some growth rate $r(T)=r_{T}$ at a later time $t=T$, then we can sum over all possible histories, or paths ${ }^{6}$, which satisfy the constraint, $\delta\left(r(t)-r_{T}\right)$. This allows the evaluation of the probability distribution function $P\left(r_{T}, T ; r_{0}, 0\right)$ for growth rates satisfying the $\delta$-function constraint. We proceed by evaluating the expectation value $E\left(\delta\left(r(t)-r_{T}\right)\right)$, i.e., 


$$
\begin{aligned}
P\left(r_{T}, T ; r_{0}, 0\right) & =E\left(\delta\left(r(t)-r_{T}\right)\right) \\
& =\int \delta\left(r(t)-r_{T}\right) d \mu \\
& =\int \delta\left(r_{0}+B^{H}-r_{T}\right) d \mu,
\end{aligned}
$$

where we used Eq. (3.1) and $d \mu$ is a Gaussian white noise measure ${ }^{7}$. Writing the delta function in terms of its Fourier representation we have,

$$
\begin{aligned}
P\left(r_{T}, T ; r_{0}, 0\right)= & \int\left(\frac{1}{2 \pi} \int_{-\infty}^{+\infty} \exp \left\{i k\left[\left(r_{0}-r_{T}+B^{H}\right)\right]\right\} d k\right) d \mu \\
= & \frac{1}{2 \pi} \int_{-\infty}^{+\infty} d k \exp \left\{i k\left[\left(r_{0}-r_{T}\right)\right]\right\} \\
& \times \int \exp \left\{i k \int_{0}^{T} \frac{\left(T-t^{\prime}\right)^{H-\frac{1}{2}} \omega\left(t^{\prime}\right) d t^{\prime}}{\Gamma\left(H+\frac{1}{2}\right)}\right\} d \mu .
\end{aligned}
$$

The integration over $\omega$ appears as a Fourier transform of $d \mu$, i.e.,

$$
\begin{aligned}
\int \exp \left\{i k \int_{0}^{T} \frac{\left(T-t^{\prime}\right)^{H-\frac{1}{2}} \omega\left(t^{\prime}\right) d t^{\prime}}{\Gamma\left(H+\frac{1}{2}\right)}\right\} d \mu & =\int \exp \left\{i \int_{0}^{T} f\left(t^{\prime}\right) \omega\left(t^{\prime}\right) d t^{\prime}\right\} d \mu \\
& =\exp \left\{-\frac{1}{2} \int_{0}^{T} f\left(t^{\prime}\right)^{2} d t^{\prime}\right\}
\end{aligned}
$$

where the right-hand-side is referred to as a characteristic functional ${ }^{7}$ with,

$$
f\left(t^{\prime}\right)=\frac{k\left(T-t^{\prime}\right)^{H-\frac{1}{2}}}{\Gamma\left(H+\frac{1}{2}\right)} .
$$

Integrating $\int f\left(t^{\prime}\right)^{2} d t^{\prime}$ in Eq. (4.3) and using this in Eq. (4.2) we have,

$$
\begin{aligned}
P\left(r_{T}, T ; r_{0}, 0\right)= & \frac{1}{2 \pi} \int_{-\infty}^{+\infty} \exp \left\{i k\left[\left(r_{0}-r_{T}\right)\right]\right\} \\
& \times \exp \left\{-\frac{k^{2} T^{2 H}}{4 H \Gamma^{2}\left(H+\frac{1}{2}\right)}\right\} d k .
\end{aligned}
$$

The remaining integral is a Gaussian integral which can be evaluated to yield the closed form, 


$$
P\left(r_{T}, T ; r_{0}, 0\right)=\sqrt{\frac{H \Gamma^{2}\left(H+\frac{1}{2}\right)}{\pi T^{2 H}}} \exp \left\{-\frac{H \Gamma^{2}\left(H+\frac{1}{2}\right)\left(r_{0}-r_{T}\right)^{2}}{T^{2 H}}\right\}
$$

We note that the probability distribution function $P\left(r, s ; r_{0}, 0\right)$, with $0 \leq s \leq T$, obeys an effective Fokker-Planck equation of the form ${ }^{8}$,

$$
\frac{\partial}{\partial s} P\left(r, s ; r_{0}, 0\right)=\frac{s^{2 H-1}}{2 \Gamma^{2}\left(H+\frac{1}{2}\right)} \frac{\partial^{2}}{\partial r^{2}} P\left(r, s ; r_{0}, 0\right)
$$

Moreover, Eq. (4.7) yields the usual diffusion equation for Brownian motion when the Hurst index is $H=1 / 2$, i.e.,

$$
\frac{\partial}{\partial s} P\left(r, s ; r_{0}, 0\right)=\frac{1}{2} \frac{\partial^{2}}{\partial r^{2}} P\left(r, s ; r_{0}, 0\right)
$$

\section{Scaling Property}

The probability distribution function $P\left(r_{T}, T ; r_{0}, 0\right)$ is known to possess a selfsimilarity property ${ }^{9}$ which facilitates our investigation of scaling that could manifest in complex systems. We first simplify Eq. (4.6) by defining, $x=r_{0}-r_{T}$, and $D=1 / 4 H \Gamma^{2}(H+1 / 2)$, and writing $P\left(r_{T}, T ; r_{0}, 0\right)=P(x, T)$ as,

$$
P(x, T)=\sqrt{\frac{1}{4 \pi D T^{2 H}}} \exp \left\{-\frac{x^{2}}{4 D T^{2 H}}\right\} .
$$

If we apply the scale transformation,

$$
x \rightarrow \kappa^{-1} x \quad ; \quad T \rightarrow \kappa^{-1 / H} T
$$

for a given constant $\kappa$, we obtain

$$
P\left(\kappa^{-1} x, \kappa^{-1 / H} T\right)=\kappa P(x, T) .
$$

Equation (5.3) shows self-similar behavior of the probability distribution function.

The Laplace transform $\mathcal{L}[\bullet]$ of the scaled probability distribution function $P\left(\kappa^{-1} x, \kappa^{-1 / H} T\right)$, Eq. (5.3), can also be evaluated. This will be useful in comparing the present formalism with actual data. We have,

$$
\mathcal{L}\left[P\left(\kappa^{-1} x, \kappa^{-1 / H} T\right)\right]=\frac{1}{2} \sqrt{\frac{\kappa}{D}} \exp \left\{-\sqrt{\frac{\kappa}{D}}|x|\right\}
$$




\section{Comparison with Empirical Data}

The result Eq. (5.4) of the previous section can directly be compared with available empirical data if the width of the probability distribution, or standard deviation $\sigma$ is identified as, $\sigma=\sqrt{2 D / \kappa}$, and the starting point of the fractional Brownian motion as $r_{0}=\bar{r}$, where $\bar{r}$ is the average growth rate. Writing $|x|=|r-\bar{r}|$ where $r=r_{T}$, and $P(r)=\mathcal{L}\left[P\left(\kappa^{-1} x, \kappa^{-1 / H} T\right)\right]$, Eq. (5.4) acquires the form,

$$
P(r)=\frac{1}{\sqrt{2} \sigma} \exp \left(-\frac{\sqrt{2}|r-\bar{r}|}{\sigma}\right) .
$$

The distribution function, Eq. (6.1), has exactly the same form as the empirical formula derived from investigating complex systems. Moreover, the tent-shaped graph (Figure 1) obtained from Eq. (6.1) often manifests in many complex systems ${ }^{1,2,3,10,11,12}$. We now consider some examples.

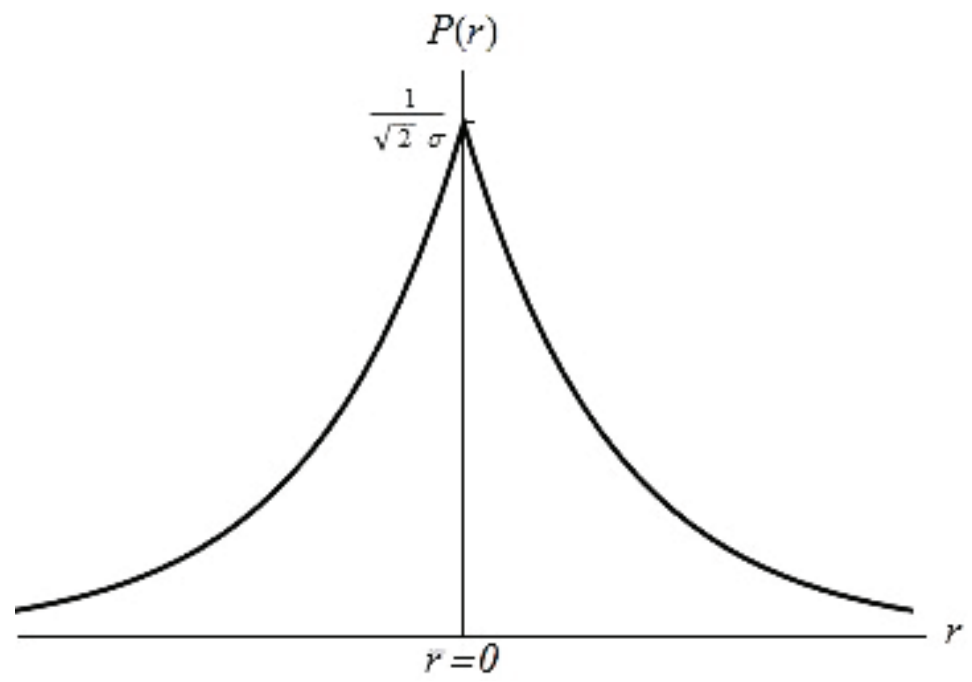

Fig. 1. Tent-shaped probability distribution function.

\subsection{Example: Growth Rates of Companies}

Our first example deals with annual growths based on data of all publicly traded US manufacturing companies producing products of all kinds between 1975 and 1991 ${ }^{1}$. Data were taken from Compustat database were all values for sales were adjusted to 1987 dollars by the GNP price deflator.

For this case, the macroscopic quantity $S$ is identified as the sales of a company. A firm's annual growth rate is then defined as, $r=\ln \left(S_{1} / S_{0}\right)$, based on the sales $S_{0}$ and $S_{1}$ for two consecutive years. Evaluating the average growth rate $\bar{r}$ and standard deviation $\sigma$ from a data set where companies have the same initial sale 
$S_{0}$, the empirically-based work of Stanley et al $^{1}$ yields a tent-shaped graph obeying the distribution function, Eq.(6.1).

\subsection{Example: Metabolic Rate Fluctuation}

In a paper by F. Labra et $\mathrm{al}^{2}$, the metabolic rate fluctuations within individual organisms was examined for 71 individuals belonging to 25 species of small terrestrial vertebrates (10 bird species, 12 small mammals, and 3 lizards). The metabolic rate $\left(\mathrm{VO}_{2}\right)$ investigated is the rate at which an animal consumes oxygen which scales with the body mass. To understand the experimental results in terms of our formalism, the macroscopic variable $S$ is identified as $S=V O_{2}$, and the $r=\ln \left(S_{1} / S_{0}\right)$ of section 2 translates into growth rate of $V O_{2}$, i.e., $r \equiv \log \left[\mathrm{VO}_{2}(t+\tau) / \mathrm{VO}_{2}(t)\right]$ where $\mathrm{VO}_{2}(t)$ and $\mathrm{VO}_{2}(t+\tau)$ are the metabolic rates observed for each specie in time intervals $t$ and $t+\tau$.

By studying the $\mathrm{VO}_{2}$ time series, F. Labra et al experimentally showed that the distributions of $\mathrm{VO}_{2}$ fluctuations of individual organisms obey a simple tent-shaped distribution as given by Figure 1 of their paper. In particular, they obtained the Laplace distribution given by Eq. (6.1) above where $\bar{r}$ and $\sigma$ correspond to the mean and standard deviation of $\mathrm{VO}_{2}$ growth rates, respectively.

\subsection{Example: Language and Fluctuations of Word Use}

Another example of a dynamic complex system is language which consists of components (words) and people interacting as users. The properties of $10^{7}$ words recorded from 1800 to 2008 in English, Spanish and Hebrew were recently analyzed and showed language independent patterns ${ }^{3}$. The huge book digitization effort of Google Inc. involving a database of words in seven languages was used as source of empirial language data. Designating $u_{i}(t)$ as the number of uses of word $i$ in year $t$, they defined the fraction of uses of word $i$ out of all word uses in the same period as,

$$
f_{i}(t) \equiv u_{i}(t) / N_{u}(t)
$$

where $N_{u}(t)$ is the total number of indistinct word uses printed in year $t$. The authors proceeded to define the growth rate as,

$$
r_{i}(t) \equiv \ln \left(\frac{f_{i}(t+\tau)}{f_{i}(t)}\right)
$$

for single year growth rates $\tau=1$. In considering the characteristic time for a word's general acceptance, the $r_{i}(t)$ was then normalized as, $r_{i}^{\prime}(\tau) \equiv r_{i}(\tau) / \sigma\left[r_{i}\right]$, where $\sigma\left[r_{i}\right]$ is the growth rate standard deviation and $\tau$ the age for each new word $i$. The probability density function $P(R)$ of $R \equiv r_{i}^{\prime}(\tau) / \sigma\left[r^{\prime}\left(\tau \mid T_{c}\right)\right]$ was evaluated where time $T_{c}$ is a threshold to distinguish words that were born in different historical eras. The empirical probability density function shown in Figure 6 of the paper by 
Petersen et $\mathrm{al}^{3}$ showed a tent-shaped distribution of the form (using the notation of Petersen et al),

$$
P(R) \equiv \frac{1}{\sqrt{2} \sigma(R)} \exp \left[-\frac{\sqrt{2}|R-\langle R\rangle|}{\sigma(R)}\right]
$$

where $\langle R\rangle$ is the average growth rate and $\sigma(R)$ the standard deviation. Clearly, Eq. (6.4) is of the same form as Eq. (6.1) obtained using fractional Brownian motion.

\section{Universality}

In the examples of complex systems given in the previous section, the various authors also showed that scaling holds where data for a wide range of parameter values collapse upon a universal curve. To obtain a universal curve, we apply the same scale transformation as Eq. (5.2), but using $2 \kappa$ instead of just $\kappa$ where Eq. (5.3) acquires a factor 2. Setting $\kappa=D$, the Laplace transform of the rescaled probability distribution $P(r)=\mathcal{L}\left[P\left((2 \kappa)^{-1} x,(2 \kappa)^{-1 / H} T\right)\right]$ acquires the form,

$$
P(r)=\exp \{-|x|\} \text {. }
$$

Equation (7.1) corresponds to the scaled probability distribution $p_{\text {scal }}=$ $\exp \left(-\left|r_{\text {scal }}\right|\right)$ from data of metabolic rate fluctuations of all species as given in Figure 2 of the work of $\mathrm{F}$. Labra et $\mathrm{al}^{2}$ showing a universal form regardless of taxonomic affiliation.

In the case of the growth of companies, the distribution function $P(r)$ becomes independent of the initial sales value $S_{0}$. All data regardless of the initial value of $S_{0}$ collapse upon a single curve as given by Figure 3 of the paper by M. H. Stanley et $\mathrm{al}^{1}$.

\section{Sensitivity to Changes in $\mathbf{H}$}

In complex systems, there is an observed scaling relation between the standard deviation of growth rates $\sigma$ and the quantity $S$ whose growth fluctuation is being studied. In particular, one has ${ }^{1,2,3}$,

$$
\sigma=a S_{0}^{-\beta}
$$

where $a$ is a constant (note that $\beta$ in Reference 2 is defined as $-\beta$ in References 1 and 3 ). In the present formalism, however, we obtained an analytical form for the standard deviation given by, $\sigma=1 / \sqrt{2 \kappa H} \Gamma(H+1 / 2)$. From this and Eq. (8.1), we get an expression for the initial value of $S$ in terms of the Hurst index $H$, i.e.,

$$
S_{0}=\left[a \Gamma\left(H+\frac{1}{2}\right)(2 \kappa H)^{1 / 2}\right]^{1 / \beta} .
$$


Since short and long-term memory are characterized by the Hurst index $H$, we can look at changes in the quantity $S_{0}$ as $H$ goes to a higher value $H^{\prime}$. In particular, we define an improvement factor, $\gamma=H^{\prime} / H \geq 1$. The graph of $S_{0}^{\prime} / S_{0}$ versus $H$ for different values of $\gamma$ is given in Figure 2. Clearly, the straight horizontal line at the bottom of the Figure indicates that when $H=H^{\prime}$, or $\gamma=1$, then $S_{0}^{\prime}=S_{0}$, whatever the initial value of $H$. The effect of increasing improvement factor, $\gamma=H^{\prime} / H>1$, up to $\gamma=2$, shows a dramatic increase in the corresponding $S_{0}^{\prime}$ relative to $S_{0}$, especially if the initial $H$ is below $H=0.2$ (short-memory domain).

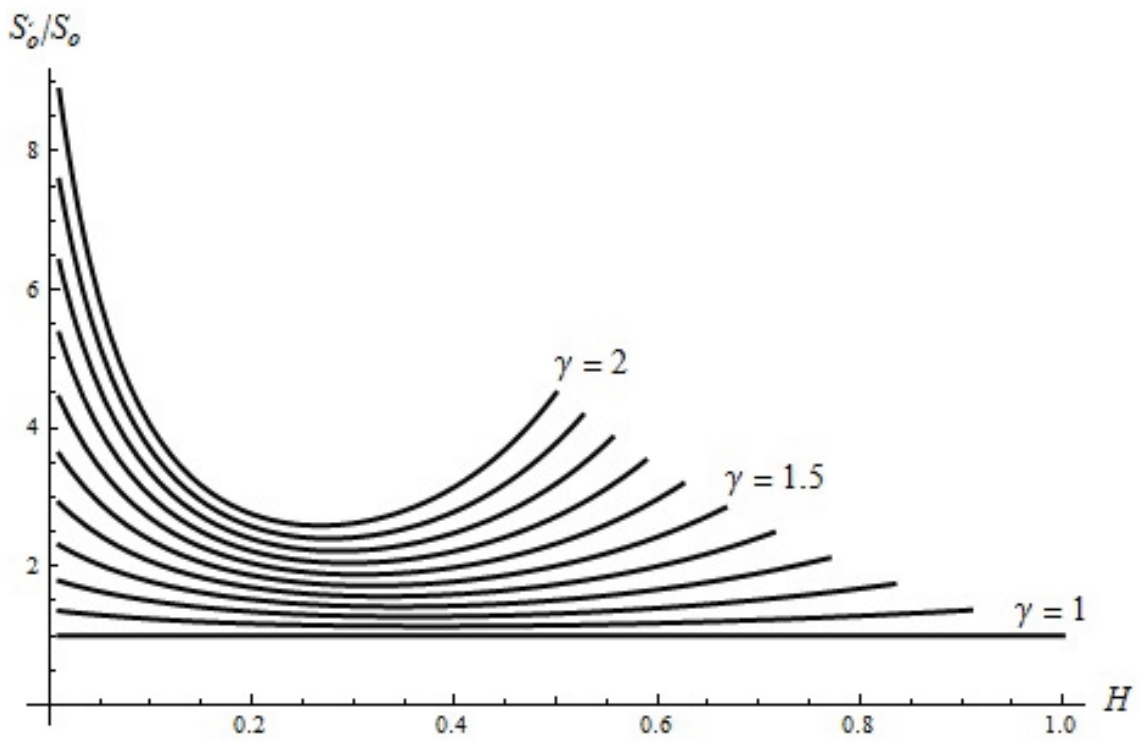

Fig. 2. Increase in sales for different improvement factors $\gamma=H^{\prime} / H$.

\section{Conclusion}

In real world situations, memory is an important feature which affects behavior in complex processes. This is exhibited in all of the examples of complex systems considered in this paper. Memory properties are incorporated in this work by using fractional Brownian motion in evaluating a probability distribution function for growth rates of macroscopic variables. We then applied the results to describe rate fluctuations with memory to characterize the evolution of complex systems that exhibit regularities and some universal features.

In evaluating the probability distribution function based on experimentally measurable quantitites for different complex systems, the width of the tent-shaped graph or standard deviation $\sigma$ plays an important role. The present analytical approach gives, $\sigma=\sqrt{2 D / \kappa}=1 / \sqrt{2 \kappa H} \Gamma(H+1 / 2)$, such that the Hurst index $H$ characterizes the diffusion evolution pattern of a complex system. The $H$ could be determined for specific situations in which the system is evolving sub-diffusively 
or in a super-diffusive manner. The determination of $H$ likewise tells us whether growth rates are positively, or negatively, correlated in time. One could, therefore, further analytically investigate long-term or short-term correlations, for instance, in the annual growth patterns of companies differing in size and in products they manufacture ${ }^{1,11,12}$, or in the metabolic rate of individuals beyond taxonomic affiliation $^{2}$, or quantify cultural memory in the use of individual words ${ }^{3}$.

\section{Acknowledgments}

The authors are grateful to the Alexander von Humboldt Foundation which made possible our visit to the Research Center BiBoS, Universität Bielefeld, and to Prof. Ludwig Streit for his hospitality and stimulating discussions on fractional Brownian motion. C. C. B. acknowledges the support of the National Academy of Science and Technology (Philippines).

\section{References}

1. M. H. R. Stanley, L. A. N. Amaral, S. V. Buldyrev, S. Havlin, H. Leschhorn, P. Maass, M. A. Salinger, and H. E. Stanley, Scaling behavior in the growth of companies, Nature 379, 804 (1996).

2. F. A. Labra, P. A. Marquet, abd F. Bozinovic, Proc. Natl. Acad. Sci. 104, 10900 (2007).

3. A. M. Petersen, J. Tenenbaum, S. Havlin, and H. E. Stanley, Sci. Rep. 2, 313 (2012).

4. R. Gibrat, Les Inégalités Economiques (Sirey, Paris, 1933).

5. Y. Mishura, Stochastic Calculus for a Fractional Brownian Motion and Related Processes, (Springer-Verlag, Berlin, 2008).

6. R. P. Feynman and A. R. Hibbs, Quantum Mechanics and Path Integrals (McGraw-Hill, New York, 1965).

7. T. Hida, H. H. Kuo, J. Potthoff, L. Streit, White Noise. An Infinite Dimensional Calculus (Kluwer, Dordrecht, 1993).

8. I. Calvo and R. Sánchez, J. Phys. A: Math. Theor. 41, 282002 (2008).

9. See, e.g., S. C. Lim and S. V. Muniandy, Phys. Rev. E 66, 021114 (2002).

10. A. M. Petersen, M. Riccaboni, H. E. Stanley, and F. Pammolli, Proc. Natl. Acad. Sci. 109, 5213 (2012).

11. G. Bottazzi, G., and A. Secchi, A., Econ. Lett. 80, 415 (2003).

12. S. Alfarano and M. Milaković, Econ. Lett. 101, 272(2008). 